Núm. 23-Julio 2020, pp. 33-45

[En línea] https://revistas.uma.es/index.php/rejienuevaepoca

\title{
DEFINICIÓN DE UN MODELO PEDAGÓGICO EN EL ÁMBITO JURÍDICO A TRAVÉS DE LA INTEGRACIÓN DE TRES FORMAS DE APRENDIZAJE-SERVICIO EN TORNO A LA SIMULACIÓN DE JUICIOS
}

Definition of a pedagogical model in the legal field through the integration of
three forms of learning-service around simulation of trials

Recibido: 9 de marzo de 2020

Aceptado: 27 de abril de 2020

\author{
Pedro Álvarez Sánchez de Movellán \\ Profesor Titular de Derecho Procesal \\ pedro.movellan@unileon.es \\ Universidad de León \\ Eva Isabel Sanjurjo Ríos \\ Profesora Contratada Doctora de Derecho Procesal \\ eva.sanjurjo@unileon.es \\ Universidad de León \\ Gracia Fernández Caballero \\ Profesora Asociada de Derecho Administrativo \\ Doctoranda en Derecho \\ gferc@unileon.es \\ Universidad de León
}

\section{RESUMEN}

Desde hace unos cuantos años, el GID "Aula Judicial de la ULE" viene trabajando con el desarrollo e innovación de las prácticas de las asignaturas de Derecho procesal, siendo siempre la simulación de juicios su actividad "estrella". Confortados por su buena acogida entre los alumnos participantes, así como por los excelentes resultados académicos arrojados, en los últimos tres cursos, hemos "rediseñado" la simulación para reconducirla hacía el fascinante mundo del "aprendizaje-servicio". Y la clave para lograrlo ha sido la implementación de tres fórmulas diferentes, pero perfectamente compatibles entre sí (ApS con personas mayores; ApS entre alumnos; y ApS extrauniversitaria), que en el presente artículo se describen pormenorizadamente. $\mathrm{Y}$ todo ello, sin desdeñar hacer una breve reseña sobre la manera en la que nos hemos visto obligados a alterar el modelo pedagógico propuesto, para así adecuarlo a la modalidad de enseñanza no presencial impuesta durante el tiempo de duración de la declaración del Estado de Alarma en nuestro país.

\section{PALABRAS CLAVES}

Aula judicial, Derecho Procesal, simulación de juicios, aprendizaje-servicio, conflictos reales, personas mayores, trabajo colaborativo, difusión. 


\section{ABSTRACT}

For a few years, the GID "Judicial Classroom of the ULE" has been working with the development and innovation of the practices of the subjects of procedural law, always being the simulation of trials its "star" activity. Comforted by its good reception among the participating students, as well as by the excellent academic results obtained, in the last three courses, we have "redesigned" the simulation to redirect it towards the fascinating world of "servicelearning". And the key to achieving this has been the implementation of three different formulas, but perfectly compatible with each other (ApS with older people; ApS between students; and extra-university ApS), which are described in detail in this article. And all this, without neglecting to make a brief review of the way in which we have been forced to alter the proposed pedagogical model, to adapt it to the non-face-to-face teaching modality imposed during the duration of the State of Alarm statement in our country.

\section{KEYWORDS}

Judicial classroom, Procedural Law, trial simulation, service-learning, real conflicts, old people, collaborative work, diffusion. 
Sumario: 1. Breve apunte sobre la propuesta innovadora y génesis de la misma. 1.1. Nuestro encuentro con el aprendizaje-servicio (ApS). 1.2. Proceso de aproximación a un Modelo de ApS. Propuestas desarrolladas. 2. Plan base para la estructuración de las propuestas. sobre la apertura progresiva de los juicios simulados como herramienta de servicio a la sociedad. 2.1. Planteamiento metodológico para las tres formas de ApS. 2.2. Enunciación de la estructura de los Juicios simulados para responder a los Modelos de ApS. 3. Definición de los objetivos pedagógicos, de aprendizaje y de servicio a conseguir. 4. Propuesta de innovación docente que integra tres formas de ApS. 4.1. Líneas de actuación que conforman la propuesta. 4.2. Aproximación a las tres formas de ApS y diferenciación entre las mismas. 4.3. Síntesis, resumen y resultados del "ApS entre alumnos" como fórmula de transición de aprendizaje-servicio. 4.3.1. Materialización del sistema de casas en la propuesta de innovación. 4.3.2. Estudio estadístico de los resultados. 4.3.3. Difusión mediática de la actividad como preparación para el "ApS extrauniversitario". 4.4 Razones para una tercera forma de ApS. El "ApS extrauniversitario". 5. Referencias globales a los beneficios evidenciados del modelo de aps y sus tres diversas formas.

\section{Breve apunte sobre la propuesta innovadora y génesis de la misma.}

\subsection{Nuestro encuentro con el aprendizaje-servicio (ApS).}

En este artículo queremos hacer una exposición de la actividad llevada a cabo por los autores firmantes en el seno de los proyectos de innovación docente propuestos, seleccionados y financiados por el Grupo de Innovación Docente "Aula Judicial" de la Universidad de León (acrónimo, GIDAJ ULE) ${ }^{1}$. La trayectoria de este grupo de innovación supera la decena entre proyectos y premios de innovación docente financiados ${ }^{2}$. Pero queremos referirnos a las actuaciones de los últimos años que nos han ido aproximando y finalmente "zambullendo" en el apasionante mundo del aprendizajeservicio (ApS).

El ApS es una manera de aprender haciendo un servicio a la comunidad. Frente a una necesidad social, los alumnos emprenden una acción de servicio a la comunidad que les ayuda a aplicar y consolidar aprendizajes en conocimientos, habilidades, actitudes, competencias, despertando su talento y poniéndolo a trabajar para el bien común ${ }^{3}$.

Es interesante cuestionarse qué es antes en el ApS: "el huevo o la gallina". En nuestro caso ha sido el aprendizaje lo que ha impulsado este camino, a través de la llamada "Aula Judicial"4 bajo cuyo paraguas hemos implementado un amplio abanico de actividades de

\footnotetext{
${ }^{1}$ Sobre la eficiente gestión de la Escuela de Formación de la ULE, de la que depende la gestión de la innovación docente se puede consultar http://servicios.unileon.es/escuela-de-formacion/.

${ }^{2}$ Nuestro GID cuenta con una amplia experiencia que se materializa en un Primer Premio a la Innovación de la Enseñanza (2004); la participación en once Proyectos financiados. También hemos aplicado el Proyecto al Curso de Formación Inicial dirigido a los alumnos de Acceso a la Carrera Fiscal en el Centro de Estudios Jurídicos (CEJ).

${ }^{3}$ Esta definición se aporta en https://aprendizajeservicio.com/, dirección en la que participa, entre otras instituciones, la Red Española de Aprendizaje-Servicio.

${ }^{4}$ En torno al nombre de Aula Judicial se creó nuestro Grupo de innovación docente, nombre que se materializó posteriormente en el blog en el que se publicitan las actuaciones llevadas a cabo por los alumnos en las actividades que describimos en el presente artículo. El mantenimiento del blog "Aula Judicial" (http://aulajudicial.blogspot.com.es/) trae razón de un Proyecto de investigación financiado por la Junta de Castilla y León. El repetido blog cuenta con unas 160.000 entradas (más de 20.000 en el último curso).
} 
innovación docente relacionadas con el Derecho procesal. Nos queremos referir aquí solamente a las últimas, que ha supuesto una aproximación progresiva $\mathrm{y}$ final desembarque en el ApS.

\subsection{Proceso de aproximación a un Modelo de ApS. Propuestas desarrolladas.}

Por poner un punto de partida, me refiero a la propuesta presentada para desarrollar en el curso 2016-2017. El título de la propuesta fue "Desarrollo y consolidación del proyecto socializador en el "Aula Judicial". Hacia una metodología colaborativa y solidaria para una enseñanza y aprendizaje de excelencia en Derecho". En aquellos momentos podríamos decir que el ApS "nos fue a visitar a casa". Realmente fue así y la visita fue en la persona de la coordinadora del Proyecto de Entrelazamiento Intergeneracional que vincula a la Gerencia de Servicios Sociales con las Universidades de Castilla y León. La diligencia y eficiencia de dicha persona (acreditada durante estos años) le llevó a buscar actividades desarrolladas en la ULE en las que pudieran participar personas mayores relacionadas con la Gerencia de Servicios Sociales. Así que en un primer momento (y con la metodología que referiremos más adelante) lo que se hizo fue incorporar a personas mayores al Aula Judicial, y más en concreto a la simulación de juicios.

Por diversas razones que expondremos entendimos que la fórmula funcionaba. Y pensamos que podía funcionar mejor. Por este motivo elaboramos una propuesta para desarrollar en el curso 2017-2018, que resultó igualmente seleccionada y financiada. Su título fue "Un "sistema de casas" para la universidad española basado en la simulación de juicios y el aprendizaje servicio. Motivación y proacción en un Aula Judicial Colaborativa". La adopción del "sistema de casas" 5 en el Aula Judicial se llevó a cabo de la siguiente manera. Se procedió a una distribución de los alumnos por casas. Las casas tienen una composición heterogénea, de manera que colaboran en la misma, alumnos de Grado en Derecho y del Máster en Abogacía. Los alumnos de $3^{\circ}$ trabajaron como Letrados Junior, los de $4^{\circ}$ Letrados Senior y los del Máster como Socios. Un sistema colaborativo vertical de conocido arraigo en los grandes despachos. De esta manera, la experiencia de ApS pasó a ser doble: de los alumnos con las personas mayores y de los alumnos de cursos superiores con los de cursos inferiores.

Por tanto, podríamos decir que en aquella propuesta del curso 2017-2018, partiendo del "Aula Judicial" y del "Acercamiento Intergeneracional", apostamos por el desarrollo de un sistema colaborativo en el que se articulasen los trabajos de alumnos de Grado en Derecho y los del Máster en Abogacía. Así, con una "segunda forma de aprendizaje servicio" como línea vertebradora, el Aula Judicial operó como un instrumento de trabajo colaborativo entre alumnos, donde los pertenecientes a cursos más avanzados asisten en su trabajo a los estudiantes noveles. Un sistema de casas que simulando la estructuración de un despacho de abogados también supone aprendizaje servicio.

Por último, en el curso 2018-2019 obtuvimos también el reconocimiento y la financiación para una nueva propuesta, "Un modelo de aprendizaje servicio en torno a la simulación de juicios. Desarrollo de un sistema colaborativo intra y extra universitario

\footnotetext{
5 El llamado "sistema de casas" es conocido como una característica vinculada a la enseñanza británica tradicional. Frente a las clásicas organizaciones de alumnos en la Universidad española el "sistema de casas" se singulariza por el trabajo colaborativo vertical, agrupando a alumnos de distintos cursos y estableciendo los cauces para la colaboración entre ellos.
} 
de atención al mayor". El planeamiento de esta última propuesta, colofón de las anteriores lo explicaremos en las siguientes páginas.

2. Plan base para la estructuración de las propuestas. sobre la apertura progresiva de los juicios simulados como herramienta de servicio a la sociedad.

\subsection{Planteamiento metodológico para las tres formas de ApS.}

a) Para la puesta en marcha del aprendizaje servicio que planteamos, el elemento clave es el desarrollo de los Juicios simulados. Y para que éstos sean una realidad, en primer lugar, será preciso proporcionar a los alumnos un conocimiento suficiente de la materia procesal, así como el manejo de conocimientos jurídicos adquiridos en el resto de asignaturas del Grado en Derecho, particularmente, de Derecho civil y Derecho penal, si bien también recientemente hemos incorporado el Derecho administrativo como se hará referencia más adelante; lo cual refleja el carácter interdisciplinar e integrador de conocimientos de nuestra propuesta, así como un buen instrumento para lograr la coordinación dentro del Título de Grado en Derecho.

A continuación, y como muestra inequívoca de que nuestra propuesta es efectivamente un proyecto de aprendizaje servicio, es preciso que el objeto del juicio simulado sea un caso real, el que plantean "personas reales" y que integran el Proyecto de Acercamiento Intergeneracional entre personas mayores y estudiantes universitarios. Dichas personas formarán parte de la simulación bien como partes del proceso (planteando el conflicto que los alumnos deben juzgar), o bien como juzgadores (siendo miembros del jurado del caso que los alumnos simulen). De esta forma, se logra motivar a los alumnos en su proceso formativo, fomentando su participación activa, al tiempo que se convierte la simulación en una herramienta más de evaluación del aprendizaje a disposición del profesorado, mejorando el seguimiento de los alumnos.

b) Continuando con la línea metodológica emprendida bajo nuestra propuesta del "Aula Judicial", que tan buenos resultados ha arrojado por resultar idónea para favorecer la motivación del alumnado, la colaboración entre ellos y la promoción del trabajo en equipo, incorporamos en un segundo momento el "sistema de casas", al que anteriormente hemos hecho mención.

El sistema de casas al que nos referimos permite la colaboración entre alumnos, donde los de cursos superiores aportan sus conocimientos y experiencias a los de cursos inferiores. Cada casa asume un rol dentro del juicio simulado que se desarrolle (demandante/acusación; demandado/defensa; tribunal), acogiendo cada una de ellas a una persona mayor, la que haya solicitado intervenir en el Proyecto y a la que representa y asiste jurídicamente. A este respecto tenemos proyectado y se pretende que algunos de los casos planteados por las personas mayores puedan ser también objeto de simulación en la actividad SIMULEON, la cual se desarrollará con motivo del encuentro nacional de Másteres de Abogacía que se ha venido celebrando en la ciudad de León durante los dos últimos cursos.

c) $\mathrm{Y}$ en un tercer momento se le da un impulso nuevo con la entrada y colaboración de los despachos de abogados, que asesorarán a los alumnos del Máster en Abogacía a fin de que, posteriormente, éstos puedan trasladar todo lo aprendido en el despacho a sus compañeros del Grado en Derecho. Se trata de que la simulación del juicio sea lo más real posible y esto se consigue en mayor grado con la intervención de los despachos, al permitir darle una perspectiva mucho más "profesional" a la relación de los alumnos con su cliente (persona mayor) y al enfoque jurídico-procesal del conflicto a simular. El 
"apadrinamiento de despachos de abogados" nos da pie a una tercera forma de aprendizaje servicio.

Para la popularización del Aula Judicial y la búsqueda de foros para conocer y enriquecer el proyecto durante el desarrollo de todas sus fases, será preciso entablar los canales de comunicación necesarios para su difusión, participando en cualquier actividad que permita la promoción de nuestra propuesta a todos los niveles sociales. En esta línea, además de numerosas apariciones en prensa, el pasado curso la radio universitaria organizó un coloquio sobre la actividad que referimos, en el que participaron alumnos, profesionales y personas mayores; con la satisfactoria casualidad de que algunos participantes fueron alumnos hace años y ahora profesionales en el desarrollo de esta actividad.

2.2. Enunciación de la estructura de los Juicios simulados para responder a los Modelos de ApS.

Partimos de tres actividades que se vinculan a cuatro asignaturas del Grado en Derecho y organizadas bajo el "sistema de casas":

a) Asignatura "Introducción al Derecho Procesal". Actividad: "Servicio social de orientación jurídica". Descripción:

Fase 1: Constitución de las casas y organización de los alumnos de la asignatura.

Fase 2: Toma de contacto con los despachos de abogados, los medios de comunicación y foros especializados. Promoción y divulgación del proyecto.

Fase 3: Entrevistas entre personas reales/mayores y alumnos.

Fase 4: Narración de conflicto.

Fase 5: Elaboración de demanda, contestación y sentencia.

Fase 6: Exposición de demanda, contestación y sentencia.

Fase 7: Divulgación del proyecto de aprendizaje servicio y sesión de valoración de resultados.

b) Asignatura "Derecho Procesal Civil". Actividad: "Aprendizaje servicio del jurista mediante la simulación de un proceso judicial civil". Descripción:

Fase 1: Constitución de las casas y organización de los alumnos de la asignatura.

Fase 2: Toma de contacto con los despachos de abogados, los medios de comunicación y foros especializados. Promoción y divulgación del proyecto.

Fase 3: Selección de conflictos expuestos por personas mayores.

Fase 4: Simulación de juicio completo en torno al mismo.

Fase 5: Celebración de juicio.

Fase 6: Reunión con la persona real/el mayor para explicarle el desarrollo del proceso en torno a su pretensión.

Fase 7: Divulgación del proyecto de aprendizaje servicio y sesión de valoración de resultados. 
c) Asignatura "Derecho Procesal Penal". Actividad: "La socialización del jurado como herramienta del jurista". Descripción:

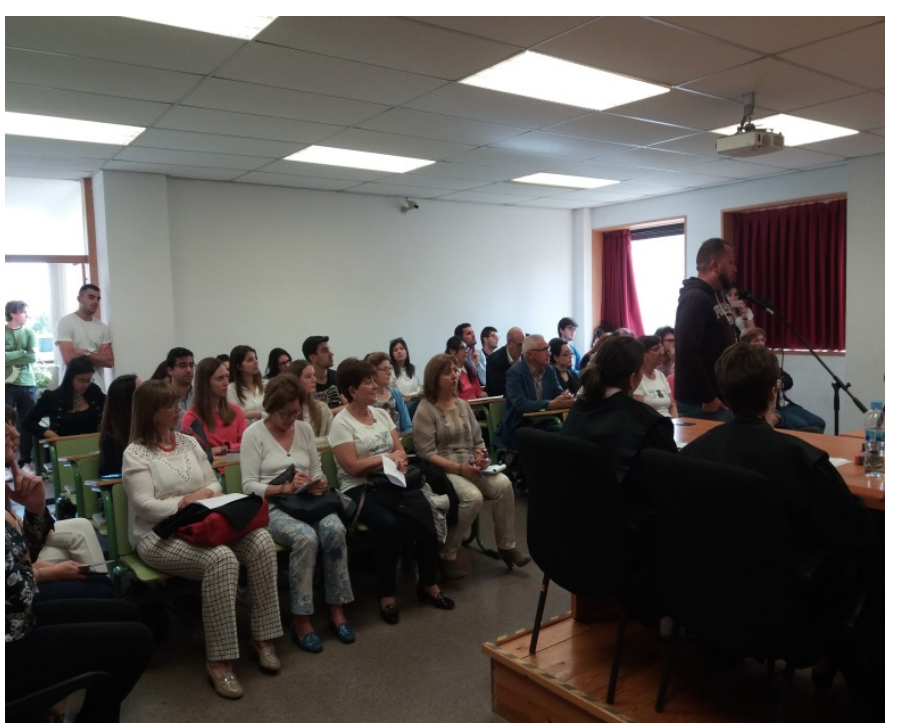

Fase 1: Constitución de las casas y organización de los alumnos de la asignatura.

Fase 2: Toma de contacto con los despachos de abogados, los medios de comunicación y foros especializados. Promoción y divulgación del proyecto.

Fase 3: Selección de un caso real instruido en los juzgados de León.

Fase 4: Elaboración de los escritos de acusación y defensa por los alumnos.

Fase 5: Exposición de los mismos a las personas mayores que serán jurados.

Fase 6: Celebración del juicio penal con jurado.

Fase 7: Veredicto de inocencia o culpabilidad por el jurado.

Fase 8: Sentencia dictada por el alumno-juez.

Fase 9: Exposición y explicación del juicio en los Centros de Mayores.

Fase 10: Divulgación del proyecto de aprendizaje servicio y sesión de valoración de resultados.

d) "Práctica de Derecho administrativo y contencioso". Actividad: "Simulación de procedimientos abreviados contencioso-administrativos con celebración de vistas mediante videoconferencia". Se precisa en este apartado que esta actividad se ha adaptado y transformado debido a la declaración del Estado de Alarma a causa del COVID-19. En este sentido, se ha podido comprobar cómo ha sido posible la realización de esta actividad empleando medios telemáticos: correo electrónico, plataforma de enseñanza online Moodle y videoconferencia. Descripción:

Fase 1: Constitución de las casas y organización de los alumnos de la asignatura, a través de Moodle.

Fase 2: Traslado a todos los alumnos de las pautas establecidas para la simulación de los casos (limitaciones formales a imagen y semejanza de los recursos de casación en materia contencioso-administrativa ante el Tribunal Supremo), a través de Moodle.

Fase 3: Traslado a los grupos demandantes de los casos para formulación de demandas (resoluciones administrativas a impugnar, y breve descripción de los hechos) a través de correo electrónico institucional.

Fase 4: Traslado a los grupos demandados de las demandas para la preparación de sus contestaciones, mediante correo electrónico institucional. En la simulación se les exige presentación de contestación escrita para valoración, de la que no se da traslado a los demandantes, pues la contestación se expondrá oralmente en el acto de la vista. 
Fase 5: Celebración de las vistas mediante videoconferencias, a través de Google Meet.

Fase 6: Dictado de las sentencias por los grupos de tribunal, y traslado a todos los alumnos de todos los materiales de la simulación, a través de Moodle.

Fase 7: Reunión de los alumnos con abogados/profesores mediante videoconferencia para analizar el desarrollo de los juicios simulados.

Fase 8: Divulgación del proyecto de aprendizaje servicio y sesión de valoración de resultados.

Acompañamos al final del artículo un cronograma del desarrollo de las actividades.

\section{Definición de los objetivos pedagógicos, de aprendizaje y de servicio a conseguir.}

La individualización de estos objetivos por categorías resulta un tanto artificial, ya que en los sistemas de ApS, el entrelazamiento entre lo pedagógico, el aprendizaje y el servicio se entrelaza. Por eso nos referimos a los diversos objetivos sin pretender "catalogarlos":

1) Consolidación de un nuevo diseño de metodologías docentes activas y renovadas. Hemos apostado por un papel particularmente protagonista para el alumno, que fuera de la "comodidad del pupitre" tendrá que relacionarse con personas mayores y profesionales de la abogacía, desarrollando sin duda su creatividad. Esta renovación metodológica, con su característica estructuración vertical del grupo y de la docencia, afecta igualmente a las tutorías curriculares y actividades teórico-prácticas.

2) La propuesta presentada supone también el diseño de nuevos procedimientos para mejorar el seguimiento de los estudiantes ya que la propia tramitación del proceso exige a alumnos y profesores ir chequeando la actividad desarrollada semana a semana.

3) A esto se acompañará un sistema de evaluación y mejora del método mediante unas encuestas detalladas a los alumnos. Introducimos un estudio estadístico completo de resultados y la presencia en foros de innovación docente.

4) La socialización del aula judicial y la implicación de despachos de abogados y del gran público nos va a exigir la presencia en los medios de comunicación y telecomunicación a través de las TICs.

5) La asignatura de Derecho procesal tiene un carácter transversal en los estudios de Derecho. Por este motivo los alumnos que participan en la propuesta presentada deben actualizar y aplicar sus conocimientos en otras materias como el Derecho civil, Derecho mercantil o Derecho penal. Todo ello con la coordinación de Grado y de Máster (entre disciplinas y Áreas) propia del "sistema de casas" descrito.

6) La implicación de la Gerencia Territorial de Servicios Sociales de la Junta de Castilla y León, de otras instituciones relacionadas con estos servicios y el contacto con los Colegios de abogados y administración corporativa supone también una coordinación con otros niveles formativos de educación, profesionales y sensibilización en temas sociales.

7) La originalidad de la propuesta unida a su proyección social invita y facilita la difusión de esta experiencia de innovación. Para ello hemos proyectado, además de 
la socialización del proyecto, la presencia en foros universitarios $y$ extrauniversitarios.

8) La propuesta responde también al objetivo de motivar y fomentar grupos estables de innovación. Nuestro grupo mantiene y renueva el aula judicial como un recurso docente eficacísimo en el ámbito de la docencia jurídica.

9) Mencionamos al final el objetivo que realmente vertebra toda la propuesta. El sistema o modelo de ApS que hemos descrito a tres niveles y con tres formas de materialización. Ya no es solo la socialización del proyecto mediante la atención a los problemas y conflictos jurídicos que padezcan las personas mayores. También se trata de un aprendizaje-servicio intrauniversitario, entre los alumnos de Grado y Máster, donde los alumnos de cursos superiores atienden a los problemas de los de cursos inferiores (que fueron sus problemas). Y extrauniversitario, con los despachos de abogados e instituciones implicadas. De esta manera refuerzan su experiencia jurídica, crecen en capacidad de servicio y enriquecen su satisfacción personal.

\section{Propuesta de innovación docente que integra tres formas de ApS.}

\subsection{Líneas de actuación que conforman la propuesta.}

Sobre las líneas propuestas por la Escuela de Formación de Profesorado de la Universidad, quisimos aplicar el proyecto en las siguientes líneas:

a) Diseño o renovación de metodologías docentes activas, que fomenten la participación del estudiante en el aprendizaje en las titulaciones oficiales de Grado y Máster. La operatividad de esta línea estaba acreditada. Efectivamente, "la necesidad agudiza el ingenio" y los alumnos de Derecho procesal no son excepción a la máxima. La participación del alumno en la defensa de un cliente en juicio es un estímulo difícilmente superable para los alumnos de últimos cursos de Derecho.

b) Diseño de procedimientos para mejorar el apoyo y el seguimiento de los estudiantes. Paradójicamente, la experiencia de "lastrar" al alumno con la responsabilidad de defender en juicio los intereses de una persona real, le da al estudiante unas alas que antes no tenía. Le permite identificar y asumir sus carencias. E incentiva al alumno para buscar el apoyo del profesor.

c) Diseño o desarrollo de métodos de evaluación del aprendizaje. La simulación de juicios es en sí misma una forma de constante evaluación. La contradicción y la necesidad de defensa de las partes va calificando en cada momento nuestros conocimientos teóricos y prácticos.

d) Coordinación dentro de un título y entre diferentes títulos universitarios. Ya hemos referido la cooperación y entrelazamiento buscada entre alumnos de Grado en Derecho y Máster en Abogacía. La práctica judicial obliga a esos alumnos de últimos cursos a repasar sus conocimientos y consultar con sus profesores de distintas áreas y materias jurídicas.

e) Aprendizaje servicio. La referimos al final por ser el hilo conductor de todas las anteriores. Y el objeto que desarrollamos en este trabajo.

\subsection{Aproximación a las tres formas de $\mathrm{ApS}$ y diferenciación entre las mismas.}

El GID “Aula Judicial de la ULE” viene trabajando con el desarrollo e innovación de las prácticas de las asignaturas de Derecho procesal. Desde la adopción del Convenio Marco de colaboración entre la Gerencia de Servicios Sociales de Castilla y León y la 
ULE, ese Aula Judicial implementa el Proyecto de Acercamiento Intergeneracional entre personas mayores y estudiantes universitarios. Y es esta una "primera forma de aprendizaje servicio", a la que nos referiremos como "ApS con personas mayores".

La "segunda forma de aprendizaje servicio", tal y como ya hemos expuesto, viene de la mano del trabajo colaborativo entre alumnos, donde los pertenecientes a cursos más avanzados asisten en su trabajo a los estudiantes noveles introduciendo de esta manera el referido "sistema de casas". Esta segunda forma pasaremos a denominarla "ApS entre alumnos".

Finalmente, en la propuesta desarrollada durante el curso 2018-2019 hemos querido introducir una "tercera forma de aprendizaje servicio": el extrauniversitario. Y en este sentido hemos buscado la implicación de despachos de abogados, diversos operadores jurídicos y del gran público (por medios de comunicación y telecomunicación) al servicio de las personas mayores y de los alumnos. A partir de ahora nos referiremos a ella como "ApS extrauniversitaria".

4.3. Síntesis, resumen y resultados del "ApS entre alumnos" como fórmula de transición de aprendizaje-servicio.

Los elementos innovadores de aquella propuesta, a los que hemos aludido anteriormente, se plantearon de la siguiente manera:

\subsubsection{Materialización del sistema de casas en la propuesta de innovación.}

El "sistema de casas" se identifica por un trabajo colaborativo singular: un sistema de educación vertical, agrupando a alumnos de distintos cursos en un grupo de trabajo o casa de composición heterogénea. Todo ello, en nuestra propuesta concreta, al servicio de la acogida al mayor, en la actividad de simulación de juicios. Tradujimos en nuestro Proyecto el "sistema de casas" de la siguiente manera:

a) Se dispuso una distribución de los alumnos por casas. Las casas debían tener una composición heterogénea, de manera que colaborasen en la misma, alumnos de Grado en Derecho y del Máster en Abogacía.

b) La distribución de alumnos del Grado se llevó a cabo por un sistema aleatorio para evitar desequilibrios entre los mismos. En cambio, la distribución de alumnos de Máster, encargados de tutelar el trabajo de los alumnos de Grado, fue selectiva, incorporando a uno u otro alumno a las casas según hayan ido manifestando a lo largo del Máster en Abogacía una actitud más solvente y profesional.

c) Teníamos planificado fomentar el sentido de pertenencia propio del "sistema de casas" y la identidad de las mismas, adoptando para ellas el nombre y el patronazgo de los grandes despachos que actúan en España. Este objetivo no hemos podido desarrollarlo en aquel curso y hemos querido incorporarlo al "ApS extrauniversitario" de este último curso.

d) Cada una de las casas se ha responsabilizado de alguna de las personas mayores que solicitaron participar en el Proyecto, asumiendo la casa y cada uno de sus miembros la defensa judicial de sus intereses. Este quizá haya sido el punto fuerte del modelo. 
La implicación de los alumnos con las personas mayores, en último extremo, es la clave del funcionamiento de las tres formas de ApS referidas.

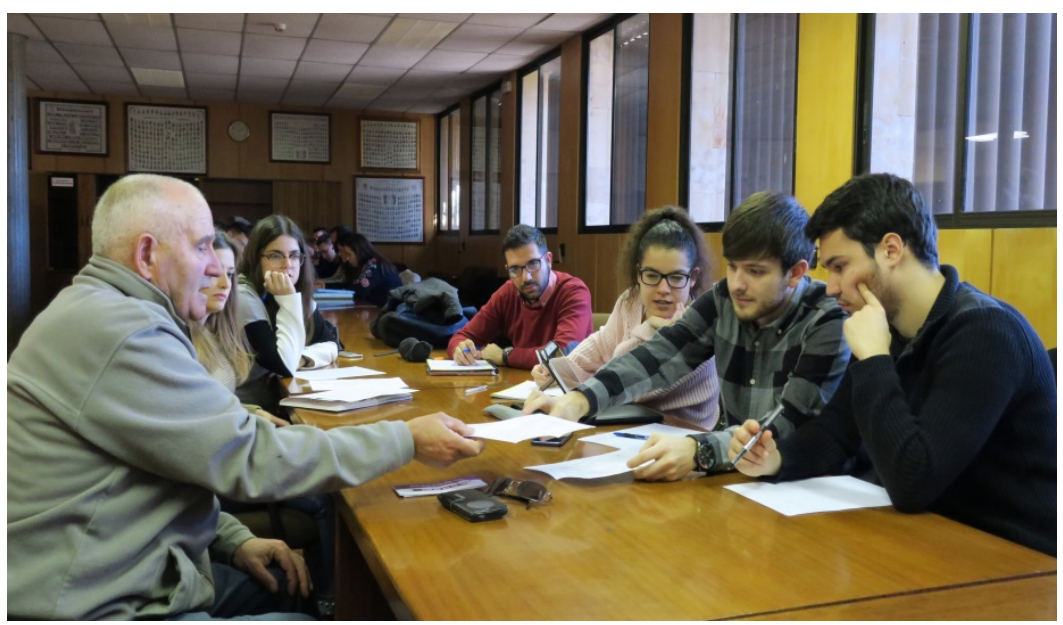

Esta actividad desplegada por las casas se ha materializado en la celebración de distintos tipos de juicios (en torno a la veintena por cada curso académico). Por otro lado, documentamos como se hace en los tribunales de justicia los expedientes correspondientes a dichos juicios, de los cuales se ha facilitado una copia a las personas mayores que han participado en la propuesta.

\subsubsection{Estudio estadístico de los resultados.}

Un segundo objetivo de la propuesta fue la realización de un estudio estadístico de los resultados de la actividad llevada a cabo. Para ello incorporamos un nuevo miembro en el Grupo de Innovación, profesor de la Escuela de Ingeniería (Área de Procesos de Fabricación). El punto de partida para la realización de esos estudios fueron las encuestas que se hicieron, diferenciadas por estamentos (personas mayores y alumnos) elaboradas en un sistema mixto de puntuación por ítems y formulación de preguntas breves. Con carácter general, la valoración de la actividad por parte de personas mayores y alumnos fue muy positiva. Y los comentarios vertidos de mucho interés de cara al desarrollo y mejora del Proyecto.

\subsubsection{Difusión mediática de la actividad como preparación para el "ApS extrauniversitario".}

En tercer lugar y por lo que respecta a la difusión mediática presentamos una variada oferta a los medios de comunicación para el seguimiento de la actividad y la presentación de resultados. De la difusión que se ha podido dar al desarrollo del Proyecto hemos recibido un eco importante y una valoración muy satisfactoria. Hicimos acopio de las noticias que aparecieron al respecto. Por referirnos solo a la prensa local podemos hacer mención a titulares como los siguientes: "Alumnos de Derecho trabajan en un proyecto de innovación docente colaborativo y de servicio"; "Estudiantes de Grado y Máster de la Abogacía elaboran una demanda simulada sobre un problema real propuesto por el Programa de Acercamiento Intergeneracional”; "Pleitos intergeneracionales"; “CCómo se declara?: aprobado"; "Entre el veredicto y la nota" ... Esta labor mediática fue realizada en colaboración con el servicio de prensa de la Universidad ${ }^{6}$.

\footnotetext{
${ }^{6}$ Acompañamos la relación de enlaces a noticias aparecidas Juicio Civil:

-http://www.unileon.es/noticias/alumnos-de-la-facultad-de-derecho-trabajan-en-un-proyecto-deinnovacion-docente-colaborativo-y-de-se
} 


\subsection{Razones para una tercera forma de ApS. El "ApS extrauniversitario".}

El Aula Judicial había implementado el Proyecto de Acercamiento Intergeneracional entre personas mayores y estudiantes universitarios. Y esto es lo que dio lugar a la "primera forma de aprendizaje servicio". Partiendo del "Aula Judicial" y del "Acercamiento Intergeneracional", apostamos por el desarrollo de un sistema colaborativo en el que se articulasen los trabajos de alumnos de Grado en Derecho y los del Máster en Abogacía. Así, con esta "segunda forma de aprendizaje servicio" como línea vertebradora, el Aula Judicial opera como un instrumento de trabajo colaborativo entre alumnos.

Para aquella propuesta teníamos proyectada y no fuimos capaces de ejecutar una complementación del sistema de casas consistente en la participación de grandes despachos en el proyecto. Básicamente se pensaba en una ocasión para que los alumnos tomasen contacto y conocimiento de la organización de los despachos de abogados, para poder analizar en la realidad eso que simulaban con el sistema de casas. La falta de resultados concluyentes de la experiencia hasta el final de curso no nos permitió este objetivo.

En la última propuesta y ante el reto de volver a intentar lo que no pudimos conseguir quisimos retomar esa idea de forma renovada. Y así introdujimos una "tercera forma de aprendizaje servicio": el extrauniversitario. En esta propuesta giramos 180 grados el objetivo de nuestra actividad de aprendizaje servicio, que hasta entonces miraba de puertas a dentro de la universidad, persiguiendo ahora su proyección fuera de las puertas de la ULE. Y en ese contexto buscamos la implicación de despachos de abogados y del gran público a través de los medios de comunicación, puestos al servicio de las personas mayores y de los alumnos.

Las personas mayores han facilitado a la ULE una experiencia de servicio a través de la docencia universitaria. Los propios miembros de la ULE (alumnos) han desarrollado un sistema de servicio a través del aprendizaje colaborativo de la organización por casas. Y a través de esta tercera propuesta buscamos la implicación extrauniversitaria en este "sistema" de aprendizaje servicio. Para lo cual nos propusimos como líneas de actuación las siguientes:

1) Apadrinamiento de despachos de abogados. Queremos explicar lo que hemos hecho. El sistema de aprendizaje servicio que hemos desarrollado, donde los universitarios trabajan en la atención jurídica a las personas mayores; y los estudiantes entre sí colaboran aportando unos la experiencia que ya tiene en beneficio de los otros. La universidad lo es plenamente cuando lo que en ella se genera llega a formar parte del provecho social. Esta idea es la que queremos transmitir para tender puentes y reforzar lazos entre universidad y sociedad.

\footnotetext{
-http://digitaldeleon.com/leon/2018/01/19/alumnos-la-facultad-derecho-trabajan-proyecto-innovaciondocente-colaborativo-servicio 24757

-http://www.ileon.com/universidad/081837/alumnos-de-derecho-trabajan-en-un-proyecto-de-innovaciondocente-colaborativo-y-de-servicio

-https://www.lanuevacronica.com/pleitos-intergeneracionales

Juicio Penal

-http://www.leonoticias.com/universidad/declara-aprobado-20180509181522-nt.html

- https://www.lanuevacronica.com/entre-el-veredicto-y-la-nota
} 
Sobre este particular recibimos una de cal y otra de arena. Nuestros intentos con despachos de ámbito nacional o internacional no dieron los frutos esperados. Pero las gestiones con despachos pequeños o medianos resultaron altamente positivas. Tanto por su participación en la iniciativa como por el alto grado de satisfacción de los abogados colaboradores.

2) Popularización del Aula Judicial. Tal y como expusimos buscamos un eco mediático de la actividad llevada a cabo. En este punto el resultado fue satisfactorio. Pero lo que buscamos no es dar noticia de unas clases singulares o unos juicios universitarios. El objetivo es dar a conocer al gran público el sistema de aprendizaje servicio que se desarrolla, informando y contando conflictos que se atienden y los juicios que se tramitan con los alumnos. El ciudadano, a través de los medios de comunicación, pasará a formar parte de una especie de "gran jurado". Y como tal, el ciudadano "puede incorporarse" a nuestro sistema de aprendizaje servicio. Además, indirectamente, pensamos que de esta manera se lanza a la sociedad un mensaje positivo, dando a conocer una singular iniciativa académica y social. De esta forma también se refuerza la imagen que la universidad tiene ante la sociedad.

3) Búsqueda de foros para dar a conocer y enriquecer el proyecto. A este respecto hemos pensado en tres posibles frentes:

a) El entorno académico de foros o congresos, de carácter nacional o internacional, en el que desarrollar, exponer y someter a la opinión de otros universitarios el proyecto, los objetivos y los resultados.

b) Las instituciones y administraciones vinculadas a la prestación de servicios sociales. Desde el momento en que la propuesta busca un servicio a determinados sectores que pueden verse beneficiados por ella, la comunicación y el diálogo con instituciones y la administración especializada en estos servicios sin duda nos reportará ideas y nuevos caminos para mejorar la experiencia.

c) Colegios de abogados y administración corporativa. Es el segmento profesional que buscamos implicar en el desarrollo de nuestra propuesta por lo que deberemos exponerles las actividades que ya hemos desarrollado y las que queremos poner en funcionamiento. De ellos esperamos además de su colaboración la propuesta de mejoras en el proyecto.

\section{Referencias globales a los beneficios evidenciados del modelo de aps y sus tres diversas formas.}

1) El beneficio mediato que legitima y justifica los demás es una mejora en el rendimiento académico del alumno. Podemos resumir la propuesta como un programa de desarrollo proactivo del alumno con su correspondiente reflejo en aprendizaje y evaluación. De las encuestas usadas estos años pensamos que estamos en el buen camino. Las realizadas por los alumnos daban una valoración de la actividad innovadora próxima al 9 sobre 10, siendo la opinión más repetida la de la utilidad de la actividad para conocer y profundizar en el Derecho Procesal.

Junto al trabajo del alumno la metodología propuesta permite un seguimiento del aprendizaje a través de los juicios. La necesidad de ir superando las diversas fases del proceso se convierte en un criterio evaluador.

2) El beneficio inmediato es la mayor motivación y participación del alumno. Lograr una transformación en el ánimo (encontrar nuevas razones para aprender) que le predisponga al aprendizaje. La proyección extrauniversitaria que proponemos 
pensamos que va a abundar en este sentido. La experiencia nos ha enseñado que el contacto con profesionales hace crecerse al alumno.

3) Estos beneficios se alcanzan tanto por la responsabilización personal en un trabajo colaborativo, tanto con las personas mayores y con agentes sociales externos, como por la actividad colaborativa fomentada por el "sistema de casas".

4) La propuesta es en sí misma una proyección hacia el exterior de la actividad innovadora. También es esencial y eficazmente pedagógica. Y su identidad dentro del ámbito del aprendizaje no se concibe fuera de las formas del servicio. Servicio al que

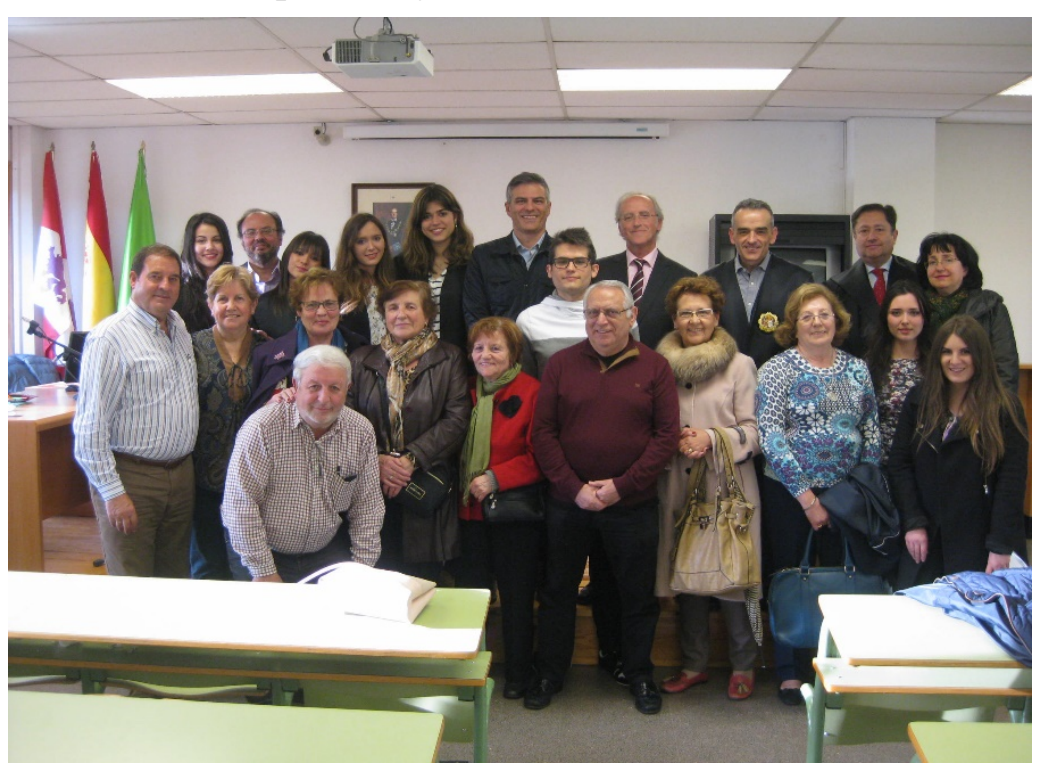
está llamada la comunidad universitaria, para formar en una dimensión académica, profesional y humana. 


\section{CRONOGRAMA DEL DESARROLLO \\ DE LAS ACTIVIDADES}

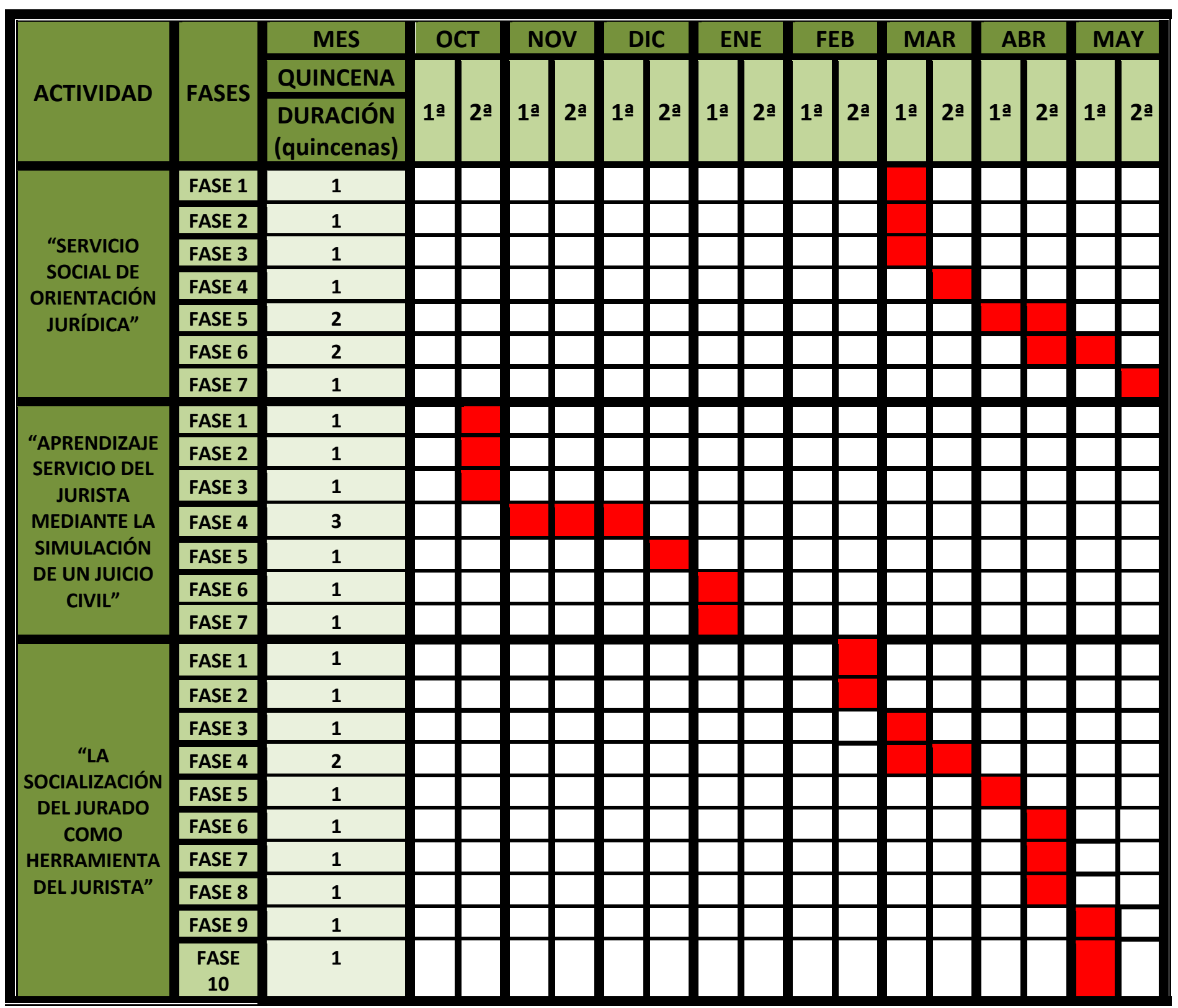

